\title{
Concise Review: How are Red Blood Cells Born, How do they Live and Die?
}

\author{
Vives Corrons J - ${ }^{2 *}$, Casafont LB $^{2}$ and Frasnedo \\ $\mathbf{E F}^{3}$ \\ ${ }^{1}$ Emeritus Professor, Red Blood Cell Pathology and \\ Hematopoietic Disorders (Rare Anaemias Unit), J osep \\ Carreras Leukaemia Research Institute, Badalona, \\ Barcelona, Spain \\ ${ }^{2}$ Professor (ret.), Polytechnic University of Catalonia, \\ Barcelona, Spain \\ ${ }^{3}$ Emeritus Professor, J osep Carreras International \\ Leukaemia Foundation, Barcelona, Spain \\ *Corresponding author: J oan-Lluis Vives Corrons, \\ Institute for Leukaemia Research, J osep Carreras Ctra \\ de Can Ruti, Camí de les Escoles s/n 08916 Badalona, \\ Barcelona, Spain
}

Received: April 05, 2021; Accepted: May 20, 2021; Published: May 27, 2021

\begin{abstract}
The average life cycle of a human RBC is approximately 120 days. Generally, by this point, the cell is worn out and damaged. RBCs pass through both the spleen and liver, where specialized immune cells called macrophages are found. Macrophages recognize when an RBC is spent, and undergo a process called phagocytosis where they digest the cell. In this process, the iron in hemoglobin is recycled for use in new blood cells and the hem molecule is degraded, conjugated to bilirubin, and eliminated from the body. All the other cellular proteins are either recycled or eliminated. Historically, this process was thought to occur exclusively in the spleen, but recent studies have shown that it occurs in the bone marrow. The RBC has been analyzed from many perspectives: cytological, hematological, and immunological, as well as from the focus of molecular biology, biophysics, and mathematics. Here we analyze how are red blood cells born and how they live and die in a brief overview of the whole process with special mention of the morphological aspects from bone marrow and spleen provided by Transmission and Scanning Electron Microscopy.
\end{abstract}

Keywords: Erythrocytes; Aging; Deformability; Rheology; Electron microscopy

\section{Introduction}

The blood and its components are one of the best investigated biological fluids due to its accessibility. Blood is the internal fluid that delivers oxygen and various metabolic substances to the cells of organs and systems in the human body, while collecting products from the cellular metabolism, and particularly carbon dioxide. It also serves as a communication fluid providing defense against outside incomes on our organism. Blood is formed by plasma and cells: the Red Blood Cells (RBCs) or erythrocytes; the White Blood Cells (WBCs) or leukocytes and the platelets or thrombocytes (Figure 1a). Humans have about 4.5 to 5 liters [1] of blood in their circulatory system, and the normal concentrations of blood cells are RBCs: 4.5 to $5.9 \times 10^{12} / 1$, WBCs: 4 to $11 \times 10^{9} / 1$, and platelets: $150-350 \times 10^{9} / 1$. The Red Blood Cell (RBC) has been analyzed from many different perspectives: cytological and morphological (optical microscopy and or without phase contrast, transmission and scanning electronic microscopy, immunological analysis, molecular and genetic analysis, rheological and physicochemical studies, and mathematics. The combination of all these perspectives, with holistic visions, always represents an advance in the amalgamation of a unique reality as seen from diverse facets.

The RBCs are performing a key role in the whole blood function that is, the oxygen transport from lungs to tissues for cell respiration. In hematology, different parameters are used to characterize the RBCs: a) their quantity or the number of cells per liter of blood; b) their size, or Mean Cellular Volume (MCV), and c) their hemoglobin content or Mean Cellular Hemoglobin $(\mathrm{MCH})$ and Mean Cellular Concentration of Hemoglobin (MCCH). $\mathrm{MCV}, \mathrm{MCH}$ and $\mathrm{MCCH}$ are known as RBC indexes and are pivotal for the morphological classification of the anemias into microcytic (low MCV), macrocytic (high MCV), and normocytic (normal MCV). However, to understand the physiopathology of Hereditary Hemolytic Anemias (HHA) due to RBC defects, the most important RBC parameter is the morphology characterized by its unique biconcave discoid shape [1]. Each discocyte has a diameter of about $7.5 \mu \mathrm{m}$, a maximum thickness of 2.0- $2.5 \mu \mathrm{m}$, a diameter/thickness ratio of $~ 3.4$, a volume, referred to as MCV of 83 to $98 \mathrm{fl}$, and a surface of about $140 \mu \mathrm{m}^{2}$ (Figure 1b).

This is not a static situation, and the shape of circulating RBCs changes permanently in normal conditions and different pathologies, facilitating the diagnosis. For many years, Scanning Electronic Microscopy (SEM) has been a useful complementary tool for exploring RBC shape because a three-dimensional image has allowed the reinterpretation of the conventional morphology observation of RBCs by MGG stained blood smears, where are seen as squashed or flattened cells on extension [2]. Due to this, SEM has allowed evaluating the real RBC shape in different pathological conditions as can be demonstrated in Hereditary Spherocytosis (HS) where RBCs are spherical (Figure 1c). This very distinctive shape of RBCs is the best demonstration that, as in many other life situations, form and function are here, inseparable elements. In the human organism, cells have perfectly adapted their shapes to perform their function in the most efficient and optimum way. Due to its discoid shape RBCs are an example of this tight relationship, and it explains how a large surface area, when compared to the volume, can allow the cell to perform its function, the interchange of oxygen $\left(\mathrm{O}_{2}\right)$, carbon dioxide $\left(\mathrm{CO}_{2}\right)$ and metabolites between plasma and cells, most efficiently.

\section{How do Red Blood Cells Born?}

The RBC production, or erythropoiesis, takes place in the bone marrow hematopoietic compartment. Continuous production of RBCs unfolds within a complex three-dimensional tissue scaffold 


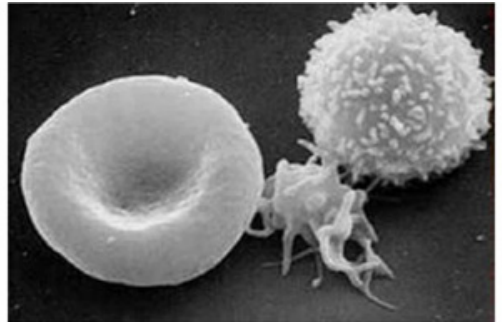

A

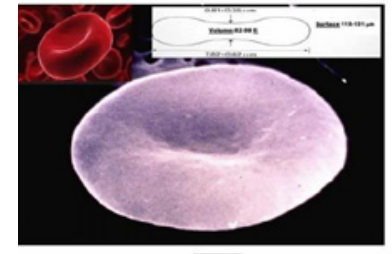

B

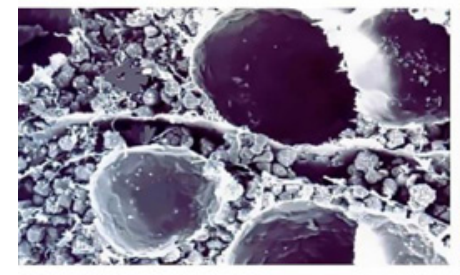

D

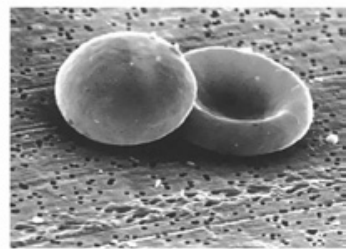

C

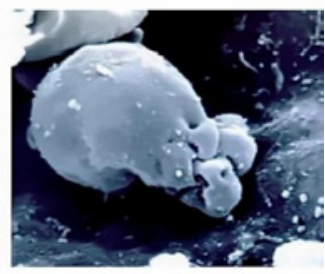

E
Figure 1: A) RBC (left) leukocyte (right) and platelet (middle) observed with Scanning Electronic Microscopy (SEM); B) RBC dimensions; C) Spherocyte (left) and normal RBC (right); D) The hematopoietic compartment of the human bone marrow where RBCs are produced. Two fat cells are observed in the bottom and upper side of the picture respectively, and a sectioned capillary in the center; E) The reticulocyte 1 (R1) is the cell that appears immediately after erythroblast nuclear extrusion. R1 is an immediate predecessor of the RBC and shows a characteristic spheroid shape with invaginated surface where the nucleolus has been extruded. The number of $\mathrm{R} 1$ in peripheral blood increases in case of stress erythropoiesis.

established by highly organized stromal cell networks of mesenchymal, neural, and vascular origin inside Bone Marrow (BM) cavities (Figure 1d). Here it is frequently seen the first stage of RBC maturation after erythroblast enucleation and is known as reticulocyte type 1 (R1). Accordingly, the $\mathrm{R} 1$ is a very young reticulocyte with a high $\mathrm{MCV}$ that still contains all the machinery necessary for the synthesis of $\mathrm{Hb}$, until its final conversion into a mature RBC. It has a sheroidal globulous shape with the surface strongly invigilated and with the roughness created by the nuclear extrusion (Figure 1e).

Collectively, stromal cells have been shown to serve two principal roles; first as primary participants of bone remodeling and metabolism, and second, as master regulators of different stages of blood cell development and production. RBC precursors are called erythroblasts; a group of cells generated from proliferating and differentiating of earlier, more immature, cells termed erythroid progenitors. Erythroid progenitors are detectable functionally only by their ability to form in vitro colonies. The development of tissue culture techniques for cloning hematopoietic progenitor cells in semisolid culture media in vitro has led to the recognition and assay in the human and murine bone marrow of at least two erythroid

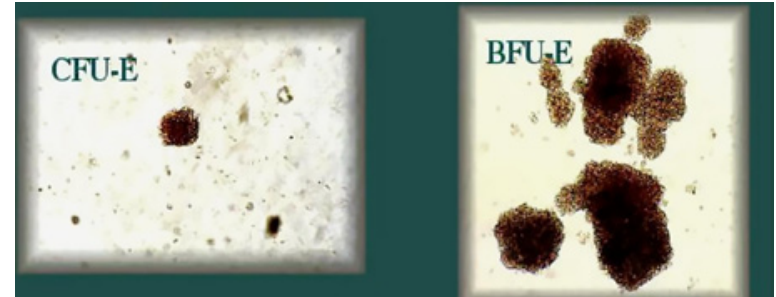

Figure 2: Erythropoietic colonies observed after in vitro bone marrow stem cell culture. BFU-E: Is the earliest erythroid progenitor (Burst Forming UnitErythroid) and erythroblasts characteristics cannot still be distinguished; CFU-E: Is the stage after the BFU-E (Colony Forming Unit-Erythroid) and erythroblasts can be seen as a stage right before hemoglobin production begins.

progenitors, the Colony-Forming Unit Erythroid (CFU-E) and the Burst-Forming Unit-Erythroid (BFU-E). Under the influence of Epo, these progenitors can grow in semisolid culture media and give rise to colonies of well-hemoglobinised erythroblasts (Figure 2). Maintenance of erythropoiesis occurs primarily through the action of Erythropoietin (Epo) promoting both progenitor proliferation and accelerating terminal maturation. A well-balanced erythropoietic mechanism is necessary for maintaining the response to a variety of normal and abnormal situations. Epo is synthesized in the kidney under the stimulus of hypoxia. Accordingly, a change of blood Hemoglobin concentration ( $\mathrm{Hb})$, leads to a change in kidney tissue oxygen, and in response to hypoxia, the kidney secretes Epo, which induces differentiation of erythroid progenitor cells, expansion of the erythroid marrow, and increased red cell production. This, in turn, leads to a compensatory increase in tissue oxygen levels [3].

The least mature recognizable erythrocyte precursor cell is known as the proerythroblast, and the various stages of maturation, in order of increasing maturity, are basophilic erythroblasts, polychromatophilic erythroblasts, and orthochromatic erythroblasts

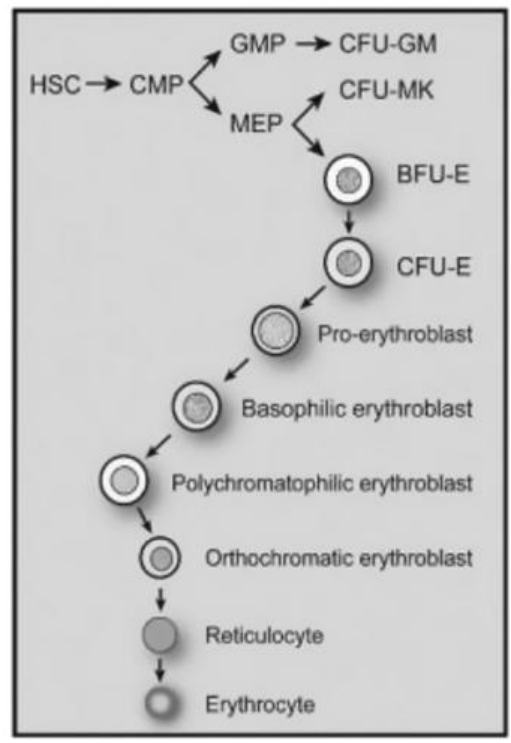

Figure 3: Maturation steps of the erythropoietic cell line. Taken from J. Quigley JG, Means RT, Glader B. Wintrobe's Clinical Hematology (13 ${ }^{\text {th }}$ Ed) 2014. 


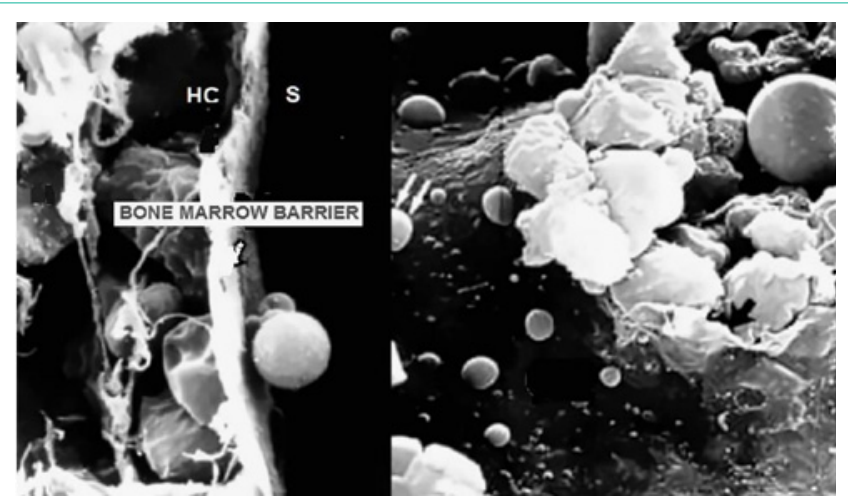

Figure 4: The blood vessels of the bone marrow constitute a barrier (Bone Marrow Barrier) that inhibit immature blood cells to leave the marrow. Only mature blood cells contain the membrane proteins, such as aquaporin and glycophorin, that are required to attach to and pass the blood vessel endothelium; Left: The "bone marrow barrier" is formed by flat endothelial cells that separates the hematopoietic compartment $(\mathrm{H})$ from the sinusoids (S). Right: RBC released into the blood stream circulation after passing the bone marrow barrier pores of 1 to $5 \mu \mathrm{m}$.

(Figure 3). Cytoplasm maturation is assessed by the change in staining characteristics, as the deep blue color derived from the high RNA content of immature cells, gives rise to the red color characteristic of hemoglobin. Nuclear maturation is evaluated by the disappearance of nucleoli and the condensation of chromatin as nuclear activity decreases. Besides, there is a gradual decrease in cell size and Epo Receptors (EpoR) expression and terminally exit from the cell cycle. Orthochromatic erythroblasts are the smallest of the nucleated erythrocyte precursors ( 8 to $12 \mu \mathrm{m}$ in diameter) and at this stage, the nucleus undergoes pyknotic degeneration, the chromatin becomes greatly condensed, and the nucleus shrinks and, in the end, it is removed from the cell by extrusion. Erythroblasts nuclear extrusion, or enucleation, occurs in orthochromatic erythroblasts producing two kinds of cells: the reticulocyte type 1 or R1 and the pyrenocyte. As mentioned before, the $\mathrm{R} 1$ is a very young reticulocyte $\mathrm{R} 1$ matures to normal reticulocyte, until its final conversion into a RBC, with its classical discocyte shape. Pyrenocytes are nucleus surrounded by a thin layer of cytoplasm and the plasma membrane and are rapidly eliminated by the macrophages of the erythroblastic island, where $\mathrm{RBC}$ membrane phosphatidylserine exposure acts as an "eat me" signal [4]. In this process, the factor of proliferation is 24 , which means that each erythroblast produces about 8 to 32 reticulocytes in approximately one week.

Cytoskeletal elements play an important role in erythroblast enucleation, acting similarly to cytokinesis but in an asymmetric way. Specifically, as observed by electron and immunofluorescence microscopy, actin filaments (F-actin) condensate behind the extruding nucleus to form the Contractile Actin Rings (CAR). Unfortunately, we are still at the beginning of understanding the mechanism and the players involved in the enucleation process.

Two events may decrease the theoretic yield of cells, one of these is the death of erythrocytes before or shortly after release from the marrow (ineffective erythropoiesis) and the second is a skipped cell division, a phenomenon that may occur with increased Epo stimulation and that results in a large hemoglobin-poor cell. These events occur only to a limited extent in normal subjects but occur
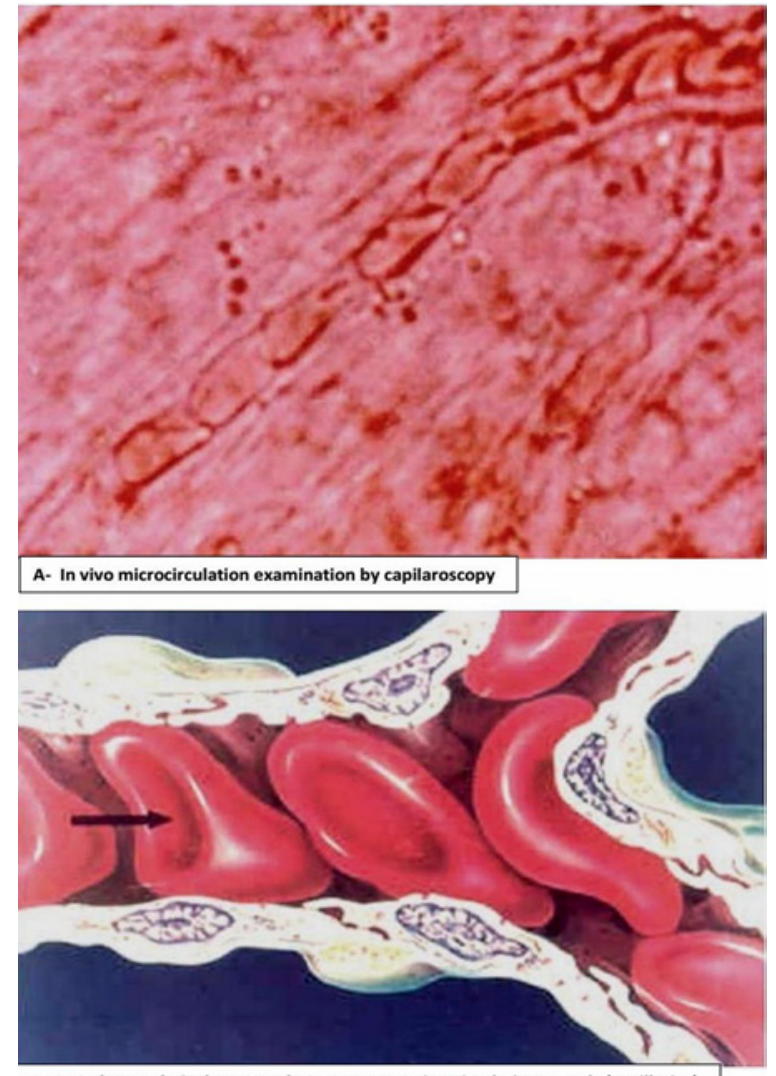

B- RBCs change their shape to adapt to narrow microcirculation vessels (capillaries)

Figure 5: A) Microcirculation is formed by arterioles with diameter sizes over $50 \mu \mathrm{m}$ and capillaries with a diameter ranging from 3 to $8 \mu \mathrm{m}$. B) RBC deformability within microcirculation passage. The RBCs have to adapt to these narrow vascular channels by changing their shape and undergoing a large deformation.

much more frequently under pathologic circumstances [5].

The mechanism of RBCs egress from the bone marrow to the bloodstream is still not yet well understood, but it seems to be determined by the so-called "bone marrow restraining barrier" that separates the hematopoietic compartment from the sinusoids. This barrier is formed by a thin layer $(0.2-0.4 \mu \mathrm{m})$ of very flat endothelial cells that completely cover the sinuses and have overlapping interdigitate and sliding joints (Figure 4). Electron microscopy has resealed that marrow sinusoids are trilaminar structures containing adventitial cells towards marrow cord, a middle basement membrane, and endothelial cells lining toward sinusoid, containing pores [6]. The adventitial cell provides an area for deformed mature RBCs, but only the pores of endothelial cells allow their egress to the bloodstream. The pores of endothelial cells are thin slots with a diameter of 1 to $5 \mu \mathrm{m}$, which is smaller than that of the RBCs, and under normal conditions, this bone marrow barrier ensures that the RBCs, released into the bloodstream have the required standard of quality [7]. The cellular movement through the barrier is very intense with some 200,000 million red blood cells, 10,000 million, granulocytes, and 400,000 million platelets passing on average each day. Several studies indicate that only mature RBCs can cross the pores of barrier membrane as compared to immature cells due to their deformability and denucleation, and it has been demonstrated that certain humoral factors 
may also increase this permeability by increasing pore size [8].

\section{How does Red Blood Cell Live?}

The RBCs have a lifetime average of about 120 days (4 months) in the bloodstream driven by the pumping force of the heart and travels more than $300 \mathrm{~km}$ providing oxygen and nutrients to all the tissue cells. A large part of their passage is through the arteries and the arterioles with diameter sizes over $50 \mu \mathrm{m}$, but also through capillaries with a diameter ranging from 3 to $8 \mu \mathrm{m}$ (Figure $5 \mathrm{a}$ ), which is less than its mean cell diameter, of about $7.5 \mu \mathrm{m}$. For the fluid traffic necessary to reach all the tissues and cells, the RBCs have to adapt to these narrow vascular channels by changing their shape and undergoing a large deformation (Figure 5b). Accordingly, deformability is the key $\mathrm{RBC}$ physical property to ensure suitable tissue oxygenation and it is the result of three geometrical and physicochemical characteristics: a) Shape (biconcave discocyte) depending on S/V ratio; b) Viscosity depending on intracellular hemoglobin concentration and its physicochemical state, and c) Viscoelasticity largely determined by the cytoskeleton an actin-spectrin network that underlies the lipid leaflet of the membrane [8]. This structure consists of long twisted strands of alpha and beta spectrin and actin filaments. Spectrin forms the inner shell of the RBC and gives the cell its deformability (Figure 6). Spectrin is bound to the lipid bilayer of the membrane at sites containing the anion exchanger, band 3 via cytoskeletal proteins, ankyrin, and adducin. Some of the transmembrane proteins (such as glycophorin A) are RBC antigens and contribute to the blood group system [9].

In the practice, the cellular shape is quantified by the ratio between the RBC surface (S) and its volume (V) or S/V ratio. Due to their discoid form, normal RBCs have an $\mathrm{S} / \mathrm{V}$ ratio of about 1.56 , indicating an excess of the membrane with regards to the volume. This allows RBCs to change their shape without increasing the surface and subsequently, to undergo highly deformable in the bloodstream. In mechanical terms, this means that RBCs can change their shape under the changes of the capillary diameter and pass through the Inter-Endothelial Slits (IES) in the spleen [10]. The spleen, is a highly vascularised organ, with a blood flow, of about $300 \mathrm{ml} / \mathrm{min}$, which represents around $6 \%$ of the cardiac flow. In humans, it plays an

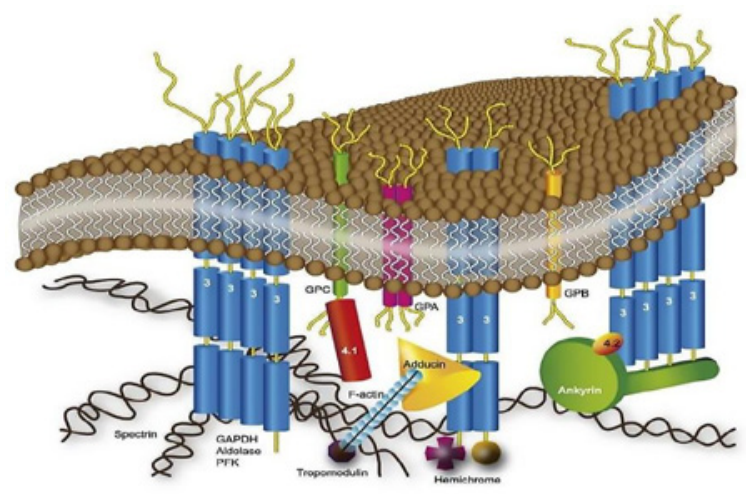

E. Pasini, H. Lutz, M. Mann, A. Thomas
Published in Journal of proteomics 2010

Red blood cell (RBC) membrane proteomics--Part l: Proteomics and RBC physiology.

Figure 6: Simplified schematic representation of the interaction between RBC membrane bilayer and the underlying cytoskeleton. important role in the immune system by protecting the body from pathogenic microorganisms reaching the bloodstream, through innate phagocytosis or adaptive responses operated by lymphocytes and antibodies. However, due to the IES, the spleen also serves as a filter that can remove RBCs from circulation when alterations in their size, shape, and deformability are detected. The spleen vasculature is composed of a complex network of capillaries, venules, arterioles, but it has a very particular and characteristic structure formed by the endothelial cells of the splenic sinuses, that anastomosed together, cover the sinuses channels without any type of junction (Figure 7). This structure allows the opening of Inter-Endothelial Passage Slots (IES) of about 0.2 to $0.4 \mu \mathrm{m}$ of diameter, and RBCs are obliged to pass through these IES (Figure 8). This serves as a filter for altered or senescent RBCs because only the cells with normal deformability can overcome this particular splenic barrier. The dynamics of passing through these narrow openings serve not only as a filter for assessing the RBC's quality but also as a test of their viability. Moreover, through this process, the spleen eliminates the RBC particles such as parasites and inclusions, thereby ensuring that only the full deformable cells may rapidly pass through the barrier posed by the splenic filter [10]. Red Blood Cells (RBCs) can be cleared from circulation when alterations in their size, shape, and deformability are detected. This function is modulated by the spleen-specific structure of the Interendothelial Slit (IES) that selects RBCs for continued circulation based on their geometry, consistent with prior in vivo observations. A companion analysis provides critical bounds relating surface area and volume for healthy RBCs beyond which the RBCs fail the "physical fitness test" to pass through the IES, supporting independent experiments [11]. Accordingly, when the RBC shape is unable to adapt to these stressing circulation conditions, it is easily trapped in the spleen and destroyed (hemolysis).

The archetypical disease where mechanical retention of RBCs in the spleen is the central pathogenic process is Hereditary Spherocytosis (HS), where the RBCs are spherical and, therefore, non-deformable (Figure 3). HS is a genetic disorder resulting in dysfunctional membrane proteins that play a role in transforming the shape of the RBC from a normal discocyte to a sphere. HS occurs at a frequency of 1 in 5,000 births in the Caucasian population and is the most common origin of hereditary intravascular and extravascular hemolysis. In HS, defects in band 3, ankyrin, and spectrin membrane proteins connecting the $\mathrm{RBC}$ membrane to the spectrin network can lead to the vesiculation of the unsupported lipid bilayer. Such vesiculation causes a gradual reduction in cell surface area by as much as $20 \%$ compared with that of a healthy RBC. This reduction,

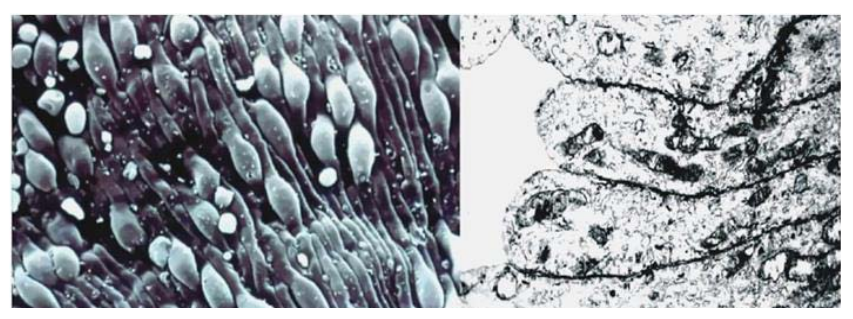

Figure 7: Wall of the spleen sinuses formed by endothelial cells that totally cover its surface without any type of junction. Right: Cross-section observed with Transmission Electron Microscopy (TEM); Left: Three dimensional views observed with Scanning Electron Microscopy (SEM). 


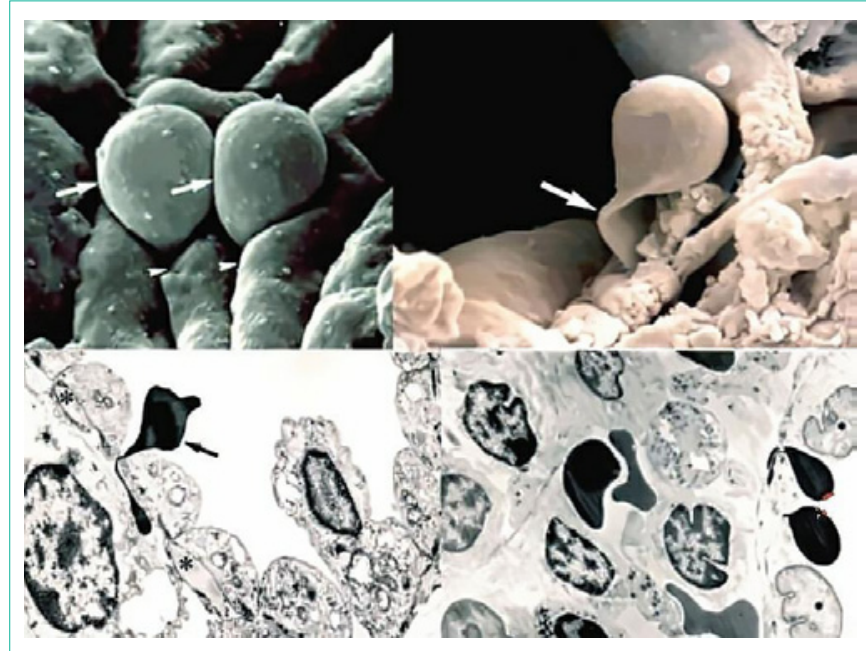

Figure 8: Passage of RBCs through the Inter-Endothelial Slots (IES) of the spleen filter. A marked deformation is appreciated by both three-dimensional view with SEM (above) and cross-section with TEM (below). The arrows indicate the RBCs passing through the narrow IES.

in turn, can significantly increase the retention rates of RBCs in the spleen because of their increased sphericity [12]. Spheres are forms that have the same surface area for a given volume and, in HS, the spherocytes have a volume of $90 \mathrm{fl}$ for a surface of about $97 \mu \mathrm{m}^{2}$, thus giving a minimum value of $\mathrm{S} / \mathrm{V}$ ratio of about 1.08 , making it impossible to change the shape without increasing its surface, and becoming rigid and non-deformable [13]. Severe reduction in RBC deformability, attributable to spherocytosis, results in the obstruction of RBCs flow as they pass through the spleen and consequently, they are phagocytosed by macrophages (Figure 9) causing hemolytic anemia and enlarged spleen (splenomegaly). The fact that surgical removal of the spleen (splenectomy) alleviates anemia to a large extent in patients with severe HS, lends support to the argument that recognition of altered deformability of RBCs is a specific function of the spleen.

\section{How do Red Blood Cells Die?}

After their hazardous, day to day, bloodstream circulation, and continuous deformability verification by the spleen, during the approximately 120 days of life the RBCs, begin to age. The whole process of RBC aging is characterized by the expression of death signaling pathways after several progressive biochemical changes mainly due to a reduction of metabolic machinery involving membrane vesiculation hemoglobin modifications and progressive failure of both cellular homeostasis and antioxidant defenses [14]. However, although the RBCs have already been used as a model for aging study, the molecular participants, as well as the signaling pathways involved, are not yet completely clarified. It seems that at the terminal stage of the aging process a cumulative cascade of events appears shortly before RBCs removal by the macrophages [15]. These events include the increase in RBC density, the nonenzymatic glycation of $\mathrm{Hb}$, and the deamidation of protein $4.1 \mathrm{~b}$ to $4.1 \mathrm{a}$ that have been widely used as sensitive $\mathrm{RBC}$ age markers. To the current knowledge, the "RBC aging phenotype", namely the repertoire of age- dependent alterations, can be safely associated with a reported decline in metabolic activity, a progressive cell shape

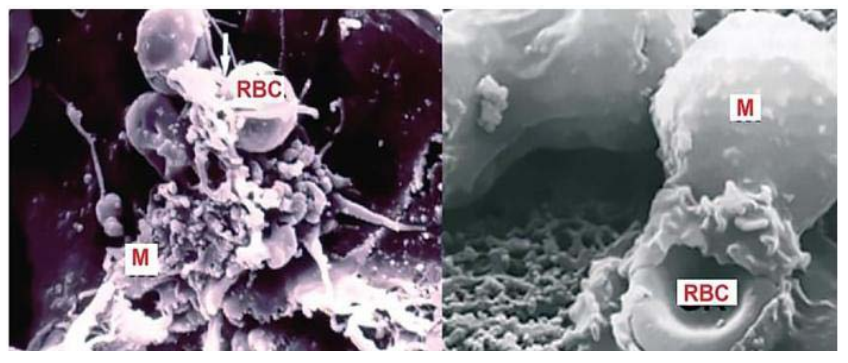

Figure 9: Death of RBCs in the bone marrow phagocytised by Macrophages (M) observed with SEM. RBCs death takes place mainly in the bone marrow, the same place where they have born.

transformation, a membrane remodeling, as well as with oxidative injury, microvesiculation, and exposure of surface removal markers. The only common feature of all these modifications is that, directly or indirectly, triggers erythrophagocytosis through the oxidant stress, common to all cell aging, and its consequence, the decrease of RBC deformability.

The decrease of RBC deformability is mainly due to skeletal architecture and remodeling of the $\mathrm{RBC}$ membrane as a consequence of ageing and the current available methods to study such parameter include rotational viscometers [16] and ektacytometry [17]. However, these techniques do not take into consideration the heterogeneity or size differences within the sample population of RBCs. For this reason, flow cytometry has become a very useful tool in the screening of membranopathies and different approaches have been described that are intended to calculate the osmotic fragility of diseased RBCs or to measure Eosin-5'-Maleimide (EMA) binding [18]. The precision of flow cytometry measurements for non- spherical cells such as RBCs is affected by the orientation of cells as they pass through a light source. This is especially true for light scatter measurements, where nearly identically shaped cells can have significantly different scatter signals owing to the variation in the cross section of each individual cell facing a focused laser source. For RBCs this variation is further complicated by secondary light scattering effects arising from their unusual bi-concave disc shape [19]. It is known that distortions of the normal $\mathrm{RBC}$ bi-concave disc shape can be the consequence of aging defects in the RBC membrane skeletal architecture and new approaches to light-scattering based on the measurement of RBC orientation have been developed An example is the new generation acustophoretic orientation measurement of RBCs that may become a potentially useful tool for the more precise measurement of the effects of RBC storage and ageing [20].

The "eat me" signal of this event is thought to be the translocation of phosphatidylserine from the inner to the outer membrane leaflet due to activation of the scramblase, while the flippase is inactivated [4] and both processes are triggered by an increased intracellular $\mathrm{Ca}^{2+}$ concentration [21] This aging progress is accompanied by the changes of many RBC surface antigens that facilitate its phagocytosis by the macrophages of the bone marrow (Figure 9). Accordingly, RBCs die in the bone marrow, the same place where they have been born, and its structural components are recycled to produce new RBCs in a splendid form of renovation.

In hereditary Hemolytic Anemias (HHA) due to RBC defects, 
cell deformability is almost always impaired, and due to the spleen barrier, the majority of abnormal RBCs are retained and destroyed by phagocytosis. As mentioned before, the classical example of this situation is HS, which can be considered an exacerbation of what happens in a normal situation when RBCs are approaching the end of their hazardous 120 days of life. This process is continuously compensated by an active RBC renewal through erythropoiesis, and, for this reason, and on account of its accessibility, blood, and its components, are one of the best biological fluids for investigating the cell aging process in normal and pathological conditions Homeostatic mechanisms must continuously adjust the balance between RBC production and destruction to maintain an appropriate number of circulating RBCs. Whereas, as mentioned before, the control of RBC production by Epo has a well- known mechanism [22], the intrinsic mechanism of underlying changes in the RBCs life span that lead to their destruction, remains largely unknown. It has been postulated that Phosphatidylserine (PS) and CD47 are the key determinants of RBC phagocytosis [23,24] and, more recently, it has been demonstrated that the quantitative aspects of these dynamics are related to oxygen homeostasis [25]. Specifically, it is suggested that PS and CD47 define a molecular algorithm that sets the timing of $\mathrm{RBC}$ phagocytosis. Significant changes in RBC lifespan, described in the literature, can be explained as alternative outcomes of this algorithm when it is executed in different conditions of oxygen availability. This theoretical model provides a unified framework to understand a variety of empirical observations regarding $\mathrm{RBC}$ biology and highlights the role of RBC lifespan as a key element of RBC homeostasis. Accordingly, this statement is based on two main assumptions. First, that the pattern of PS and CD47 expression changes during the life of the cell, as evidenced by differences between young and aged RBCs, and second, that the conditions that trigger RBC phagocytosis differ in the subsequent behavior they elicit on macrophages. Probably, the combined effects of accelerated PS expression and the lower CD47 expression may induce an immune response against host RBCs with the production of anti-RBC antibodies that would result in a shortened RBC lifespan. Here Epo levels, depending on the levels of oxygen, might continuously adjust the value of the phagocytosis thresholds, thus fine-tuning the lifespan of circulating RBCs. It should be mentioned that the high levels of Oxidative Stress (OS) might also shorten RBC life span by accelerating the rate of PS exposure in the outer membrane of the cell. And that Hypoxia-Induced Factors (HIFs) might be involved in shortening RBC life span in newly young RBCs due to their lower content in catalase activity [26]. All these mechanisms are independent and might be acting simultaneously, and in a coordinated way, to determine the RBC lifespan. However, further research is needed to unveil all the mechanisms underlying $\mathrm{RBC}$ lifespan determination.

In future publications of this section, our research group will try to provide a holistic vision of a combination of these procedures for a better understanding of RBC senescence in normal and pathological conditions.

\section{Disclosure}

The authors have no other relevant affiliations or financial involvement with any organization or entity with a financial interest in or financial conflict with the subject matter or materials discussed in the manuscript apart from those disclosed.

\section{Acknowledgement}

We are indebted to the European Commission for the Equality Plus (Erasmus+) Grant (Ref. 2019-1-TR01-KA202-076789) and to Mrs. Elena Krishnevskaya for her outstanding collaboration in rare anaemias research and in conditional support for the preparation of this manuscript.

Electron Microscopy Studies were performed in the "Escola Professional d'Hematologia Farreras Valenti" (University of Barcelona) under the leadership of Prof. Ciril Rozman Borstnar (Hemorheology Theoretical Basis and Clinical Applications 1983).

\section{References}

1. Vives Corrons JL. The Rare Anaemias. Chapter of the Rare Diseases. Intechopen. 2019.

2. Berga L, Feliu E, Ferran MJ, Rozman C. Contribution of Scanning Electron Microscopy to the structural study of human bone marrow. Sangre. 1987 27: 3939.

3. Quigley JG, Means RT, Glader B. The Birth, Life, and Death of Red Blood Cells: Erythropoiesis, The Mature Red Blood Cell, and Cell Destruction. In JP Greer, DA Arber, B Glader, AF List, RT Means, Jr., F Paraskevas, and J Foerster (Eds.), Wintrobe's Clinical Hematology (13 ${ }^{\text {th }}$ ed. pp.83-124). Philadelphia Philadelphia, PA: Lippincott Williams \& Wilkins. 2014.

4. Yoshida $\mathrm{H}$, Kawane $\mathrm{K}$, Koike $\mathrm{M}$, Mori $\mathrm{Y}$, Uchiyama $\mathrm{Y}$ and Nagata $\mathrm{S}$. Phosphatidylserine-dependent engulfment by macrophages of nuclei from erythroid precursor cells. Nature. 2005; 437: 754

5. Liang R, Ghaffari S. Advances in understanding the mechanisms of erythropoiesis in homeostasis and disease. Br J Haematol. 2016; 174: 661673.

6. Weiss L. The structure of bone marrow: functional interrelationships of vascular and hemopoietic compartments in experimental hemolytic anemia: an electron microscopic study. J. Morph. 1965; 117: 467.

7. DeBrulyin PPHS, Michelson TB, Thomas. The migration of blood cells of the bone marrow through the sinusoidal wall. J Morphol. 1971; 133: 417.

8. Berga L, Vives Corrons JL, Feliu E, Woessner S, Rozman C. Hemorheology. Theoretical Basis and Clinical Applications. Salvat Editores (Barcelona). 1983.

9. Lux S. Anatomy of the red blood cell membrane skeleton: Unanswered questions. Blood. 2016; 12: 187.

10. Mebius RE, Kraal G. Structure and function of the spleen. Nat Rev Immunol. 2005; 5: 606

11. Pivkin IV, Peng Z, Karniadakis GE, Buffet PA, Dao M, and Suresh S. Biomechanics of red blood cells in human spleen and consequences for physiology and disease. Proc Natl Acad Sci USA. 2016; 113: 7804.

12. Suresh S. Mechanical response of human red blood cells in health and disease: Some structure-property-function relationships. J Mater Res. 2006; 21: 1871.

13. Berga L. Spleen and hemolysis. Medicina Clínica (Barcelona). 1985; 85: 273.

14. Brovelli A, Minetti G. Red Cell Ageing. In: Bernhardt I, Ellory JC. (eds) Red Cell Membrane Transport in Health and Disease. Springer, Berlin, Heidelberg. 2003

15. Franco RS. The measurement and importance of red cell survival. Am J Hematol. 2009; 84: 109

16. Groner W, Mohandas N \& Bessis M. New optical technique for measuring erythrocyte deformability with the ektacytometer. Clin Chem. 1980; 26: 1435.

17. Lazarova E, Gulbis B, Oirschot BV \& van Wijk R. Next-generation osmotic gradient ektacytometry for the diagnosis of hereditary spherocytosis: 
interlaboratory method validation and experience. Clin Chem Lab Med. 2017; 55: 394.

18. King MJ, Behrens J, Rogers C, Flynn C, Greenwood D, Chamberset. Rapid flow cytometric test for the diagnosis of membrane cytoskeleton-associated haemolytic anaemia. $\mathrm{Br} J$ Haematol. 2000; 111: 924.

19. Merola F, Barroso A, Miccio L, Memmolo P, Mugnano M, Ferraro P, et al. Biolens behavior of RBCs under optically-induced mechanical stress. Cytometry A. 2017; 91: 527

20. Rico LG, Juncà J, Ward MD, Bradford JA, Bardina J, Petriz J. Acoustophoretic Orientation of Red Blood Cells for Diagnosis of Red Cell Health and Pathology. Scientific Reports. 2018; 8: 15705.

21. Bernhard I, Nguyen DB, Wesseling MC, Kaestner L. Intracellular $\mathrm{Ca}^{2+}$ Concentration and Phosphatidylserine Exposure in Healthy Human Erythrocytes in Dependence on in vivo Cell Age. Front Physiol. 2020; 10; 1629.
22. Palis J, Segel GB. Developmental biology of erythropoiesis. Blood Rev. 1998; 12: 106.

23. Bosman GJCGM, Lasonder E, Groenen-Döpp YAM, Willekens FLA, Werre JM, Novotý VMJ. Comparative proteomics of erythrocyte aging in vivo and in vitro. J. Proteom. 2010; 73: 396.

24. Freikman I, Fibach E. Distribution and shedding of the membrane phosphatidylserine during maturation and aging of erythroid cells. Biochim. Biophys. Acta. 2011; 1808: 2773.

25. Fernandez Arias C, Fernandez Arias C. How to do red blood cells. know when to die? R. Soc. Open Sci. 2017; 4: 160850

26. Song J, Yoon D, Christensen RD, Horvathova M, Thiagarajan P, Prchal JT. HIF-mediated increased ROS from reduced mitophagy and decreased catalase causes neocytolysis. J. Mol. Med. 2015; 93: 857 